Hiroki Matsuura, MD

Department of General Internal Medicine,

Okayama City Hospital; Department of General Internal Medicine, Okayama City Senoo Hospital,

Okayama, Japan
Takashi Katayama, MD

Department of Internal Medicine, Okayama City

Senoo Hospital, Okayama, Japan
Kentaro Deguchi, MD, PhD

Department of Neurology, Okayama City Hospital, Okayama, Japan

\title{
Metronidazole-induced encephalopathy: Symmetrical hyperintensity on imaging
}

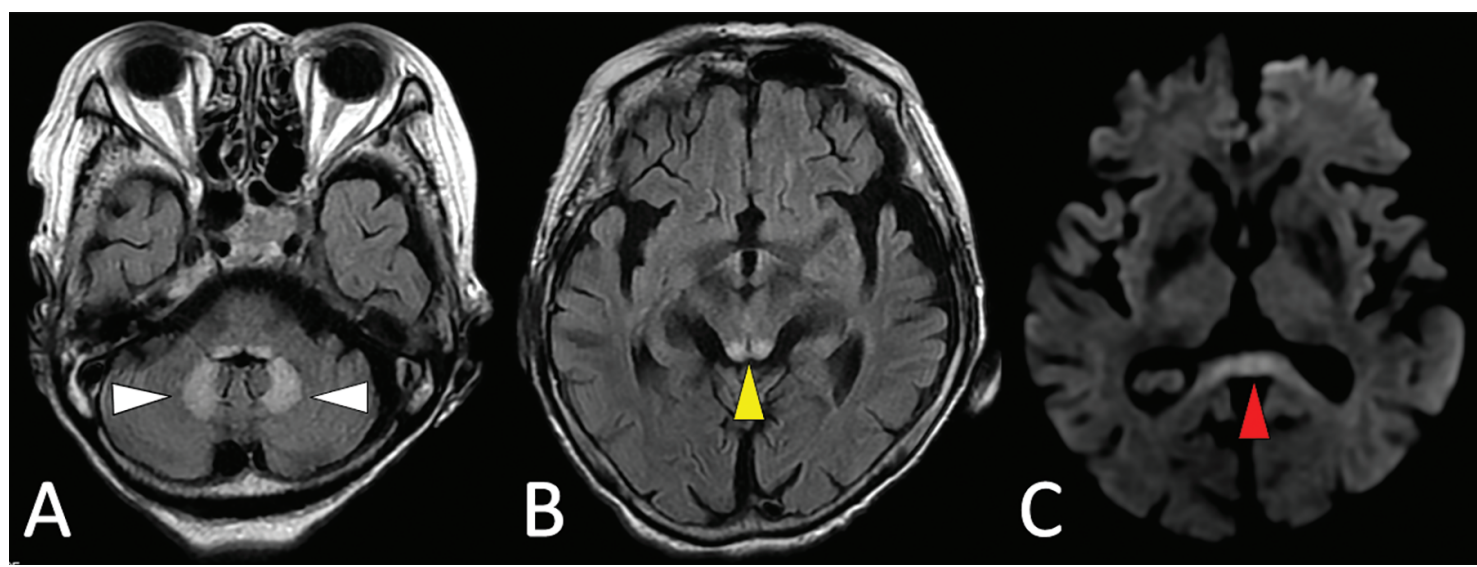

Figure 1. (A) T2 fluid-attenuated inversion recovery (T2-FLAIR) on MRI shows typical symmetrical hyperintensity in the dentate nuclei of the cerebellum, reflecting vasogenic edema (white arrowheads). (B) T2-FLAIR MRI shows hyperintensity in the tectum of the midbrain (yellow arrowhead). (C) Diffusion-weighted MRI shows hyperintensity in the splenium of the corpus callosum, indicating restricted diffusion or cytotoxic edema (red arrowhead).

A n 83-year-old woman with mild hypertension and hypothyroidism was admitted to our hospital with acute pyelonephritis. She was treated with ampicillin plus sulbactam for 10 days with a good response while she was under rehabilitation receiving physical and speech therapy. However, she suddenly experienced fever, abdominal pain, and severe watery diarrhea. A stool sample was positive for Clostridioides difficile ( $\mathrm{C}$ difficile) antigen and toxins, and she was prescribed metronidazole 1,500 mg/day for 4 weeks for gastrointestinal symptoms due to $\mathrm{C}$ difficile infection

Although the patient's gastrointestinal symptoms improved, she later presented with an acute onset of dysphagia, nausea, vomiting, dizziness, and progressively altered mental status. Her vital signs were stable. Physical findings revealed no nuchal rigidity or meningeal irritation. However, neurologic examination doi:10.3949/ccjm.88a.21007 showed dysarthria, minimal horizontal nystagmus, and unsteady gait. Results of laboratory testing were unremarkable.

Computed tomography showed moderate atrophy without cerebral bleeding. Magnetic resonance imaging (MRI) showed symmetrical T2-hyperintensity in the tectum of the midbrain, pontine tegmentum, and dentate nuclei indicating parenchymal vasogenic edema (Figure 1). Based on the characteristic imaging finding and the clinical history, we made a diagnosis of metronidazole-induced encephalopathy and immediately stopped the metronidazole therapy. After metronidazole was stopped, her neurologic symptoms improved gradually without remission.

\section{METRONIDAZOLE AND NEUROTOXICITY}

Metronidazole-induced encephalopathy is a relatively rare central nervous system disorder,
After metronidazole was stopped, her neurologic symptoms improved gradually, without remission 
associated with prolonged duration and high cumulative doses of metronidazole. ${ }^{1}$ Metronidazole is commonly used to treat a wide variety of infection-associated diseases, including pelvic inflammatory disease, bacterial vaginosis, intra-abdominal abscess, amebiasis, giardiasis, and $\mathrm{C}$ difficile. However, metronidazole has been reported to be inferior to vancomycin particularly for patients with severe cases of $\mathrm{C}$ difficile infection. ${ }^{2}$

The mechanism of neurotoxicity due to metronidazole is unknown. It is thought that metabolites of metronidazole may bind to ribonucleic acid and interfere with ribonucleic acid protein synthesis, which can lead to axonal degeneration. ${ }^{3}$ Neurologic symptoms of metronidazole-induced encephalopathy vary widely among individual patients but can include cognitive deterioration, peripheral neuropathy, weakness, dizziness, vertigo, nausea, vomiting, headache, sensory loss, and seizures. $^{4}$
A characteristic MRI finding in patients with metronidazole-induced encephalopathy is bilateral involvement of the cerebellar dentate nuclei. ${ }^{5}$ However, this is also seen in other neurologic disorders such as Wernicke encephalopathy and isoniazid or methyl bromide toxicity. Thus, the definitive diagnosis should be based on a combination of the patient's clinical history, laboratory findings, and imaging results. In most cases, the encephalopathy is reversible and generally improves within a few weeks after metronidazole is stoppped. ${ }^{6}$ However, a delayed diagnosis can have progressive, irreversible consequences, including death. ${ }^{\text {? }}$ Clinicians should consider metronidazole-induced encephalopathy in a patient presenting with new psychiatric and neurologic symptoms and signs, especially in those with cerebellar symptoms who are taking metronidazole.

\section{DISCLOSURES}

The authors report no relevant financial relationships which, in the context of their contributions, could be perceived as a potential conflict of interest.

\section{REFERENCES}

1. Huang YT, Chen LA, Cheng SJ. Metronidazole-induced encephalopathy: case report and review literature. Acta Neurol Taiwan 2012; 21(2):74-78. pmid:22879116

2. Stevens VW, Nelson RE, Schwab-Daugherty EM, et al. Comparative effectiveness of vancomycin and metronidazole for the prevention of recurrence and death in patients with Clostridium difficile nfection. JAMA Intern Med 2017; 177(4): 546-553. doi:10.1001/jamainternmed.2016.9045

3. Bradley WG, Karlsson IJ, Rassol CG. Metronidazole neuropathy. Br Med J 1977; 2(6087): 610-611. doi:10.1136/bmj.2.6087.610

4. Sonthalia N, Pawar SV, Mohite AR, et al. Metronidazole-induced encephalopathy in alcoholic liver disease: a diagnostic and therapeutic challenge. J Emerg Med 2016; 51(4):e79-e83.

doi:10.1016/j.jemermed.2016.05.038
5. McKinney AM, Kieffer SA, Paylor RT, SantaCruz KS, Kendi A, Lucato L. Acute toxic leukoencephalopathy: potential for reversibility clinically and on MRI with diffusion-weighted and FLAIR imaging. AJR Am J Roentgenol 2009; 193(1):192-206. doi:10.2214/AJR.08.1176

6. Sørensen CG, Karlsson WK, Amin FM, Lindelof M. Metronidazoleinduced encephalopathy: a systematic review. J Neurol 2020; 267(1):1-13. doi:10.1007/s00415-018-9147-6

7. Groothoff MV, Hofmeijer J, Sikma MA, Meulenbelt J. Irreversible encephalopathy after treatment with high-dose intravenous metronidazole. Clin Ther 2010; 32(1):60-64. doi:10.1016/j.clinthera.2010.01.018

Address: Hiroki Matsuura, MD, Department of General Internal Medicine, Okayama City Hospital, 3-20-1, Omote-cho, Kitanagase, Okayamacity, Okayama, 700-0962, Japan; superonewex0506@yahoo.co.jp 